\title{
Instrumento para avaliação da qualidade do Time de Resposta Rápida em um hospital universitário público
}

\author{
Instrument for assessing the quality of the Rapid Response Team at a university public hospital \\ Instrumento para la evaluación de la calidad del Equipo de Respuesta Rápida \\ en un hospital universitario público
}

\section{Alexsandro Oliveira Dias', Eleine Aparecida Penha Martins', Maria do Carmo Lourenço Haddad' \\ ' Universidade Estadual de Londrina, Departamento de Enfermagem, Programa de Pós-Graduação em Enfermagem. Londrina-PR, Brasil.}

\author{
Submissão: 05-03-2013 Aprovação: 14-08-2014
}

\section{RESUMO}

O artigo descreve a construção de um questionário para avaliação da qualidade do atendimento de um Time de Resposta Rápida, em Hospital Universitário de Londrina-PR, fundamentado no modelo conceitual de Donabedian (estrutura-processoresultado). A coleta de dados ocorreu no mês de março de 2012 e o processo de adequação do questionário foi desenvolvido com a aplicação da Técnica Delphi com a participação de 15 especialistas. Ao término do estudo obteve-se um questionário com 37 enunciados, sendo alcançado índice de concordância final na pesquisa com valores superiores a $80 \%$ para todos os conceitos. Espera-se que as contribuições do grupo de especialistas tornem o instrumento confiável e seja aplicado em outros serviços semelhantes. Aplicações futuras deste instrumento poderão trazer subsídios para melhor avaliação da qualidade dos serviços de equipes de Resposta Rápida.

Descritores: Equipe Hospitalar de Resposta Rápida; Qualidade; Questionário; Hospital Universitário; Enfermagem.

\begin{abstract}
The paper describes the construction of a questionnaire to assess the quality of care of a Rapid Response Team at the University Hospital of Londrina, based on the conceptual model of Donabedian (structure-process-outcome). Data collection occurred in March 2012 and the process of adjusting the questionnaire was developed with the application of the Delphi technique involving 15 experts. At the end of the study the questionnaire contained 37 statements, achieving final compliance level higher than $80 \%$ in all concepts. It is hoped that the contributions of the expert group produce a more reliable questionnaire to be applied in other similar services. Future applications of this instrument may provide information to better assess the quality of teams of Rapid Response services.
\end{abstract}

Key words: Hospital Rapid Response Team; Quality; Instrument; University Hospital; Nursing.

\section{RESUMEN}

El artículo describe la construcción de un cuestionario para la evaluación de la calidad de la atención de un Equipo de Respuesta Rápida en un Hospital Universitario de Londrina, Paraná, basado en el modelo conceptual de Donabedian (estructura-procesoresultado). La recolección de datos ocurrió durante el mes de marzo de 2012 y el proceso de ajuste del cuestionario fue desarrollado por medio de la Técnica Delphi con la participación de 15 especialistas. Al término del estudio se obtuvo un cuestionario con 37 enunciados, alcanzándose un índice de concordancia final en la investigación con valores superiores al $80 \%$ para todos los conceptos. Se espera que las contribuciones del grupo de especialistas afiancen la confiabilidad del instrumento y que el cuestionario sea utilizado en otros servicios semejantes. Las aplicaciones futuras podrán traer subsidios para evaluar mejor la calidad de los servicios de los equipos de Respuesta Rápida.

Palabras clave: Equipo Hospitalario de Respuesta Rápida; Calidad; Cuestionario; Hospital Universitario; Enfermería. 


\section{INTRODUÇÃO}

No Brasil ocorre um crescimento do número de Times de Resposta Rápida (TRR) em hospitais terciários, pois garantir a segurança do paciente internado é hoje um dos principais desafios da gestão da qualidade na área da saúde ${ }^{(1)}$. O TRR teve início na Austrália em 1991 e consiste em uma equipe multidisciplinar com abordagem no tratamento intensivo do paciente com sinais de agravamento clínico na unidade de internação, por meio de códigos estabelecidos para acionar o serviço. É projetado para fornecer, em tempo hábil, os recursos adequados e necessários para evitar ou reduzir a probabilidade de uma piora do quadro clínico ou risco de morte iminente para o paciente ${ }^{(2)}$.

A saúde tem a extrema necessidade de buscar constantemente a excelência em suas atividades, pois um erro não resulta apenas em prejuízo, já que pode acarretar na perda de vidas. Nesse sentido, junto do desejo de melhoria contínua nos serviços, as organizações de saúde estão cada vez mais preocupadas com a qualidade em suas estruturas e $\operatorname{processos}^{(3)}$.

Assim, os serviços de saúde iniciaram o engajamento no movimento pela qualidade já existente em outras áreas de atuação. Avedis Donabedian absorveu da teoria de sistemas a noção de indicadores de estrutura, processo e resultado, que se tornaram clássicos nos estudos de avaliação da qualidade do atendimento hospitalar em saúde ${ }^{(4)}$.

O indicador estrutura corresponde à forma como a organização se apresenta em relação aos recursos físicos, humanos, materiais, equipamentos, normas, rotinas, sistema de valores e expectativas; são as características relativamente estáveis e necessárias ao processo assistencial. O processo relaciona-se à maneira como a assistência é prestada aos clientes, segundo padrões técnico-científicos estabelecidos e aceitos cientificamente. O resultado, por sua vez, corresponde às consequências das atividades realizadas nos serviços de saúde, ou pelos profissionais envolvidos ${ }^{(5)}$.

Embora exista uma série de dificuldades para avaliar a qualidade na área da saúde, há unanimidade entre os gestores de que é necessário escolher sistemas de avaliação e indicadores de desempenho institucionais adequados para apoiar a administração dos serviços e propiciar a tomada de decisão com o menor grau de incerteza possível ${ }^{(6)}$.

O movimento pela qualidade nos serviços de saúde é hoje uma necessidade incorporada à gestão entre diversas áreas inter-relacionadas, a fim de assegurar a assistência livre de riscos ao usuário. A concentração de esforços em direção aos objetivos propostos leva à melhoria contínua desta assistência, com necessidades e implicações na conscientização de toda a equipe quanto à sua importância e ao valor de suas ações. Dessa forma, comprometimento, cooperação, dedicação e aprimoramento contínuos na área da saúde deverão conduzir aos resultados desejados para o paciente e para os profissionais $^{(5)}$.

A qualidade em saúde tornou-se um imperativo, e é a marca da modernidade. Porém, para que seja alcançada, é preciso que ocorra a sistematização de todas as suas práticas e processos. O uso de instrumentos de medida na busca pela melhoria dos indicadores para o alcance da alta produtividade, humanização e baixo custo podem fornecer indicações práticas e válidas sobre o nível de qualidade da assistência prestada, e acrescentam que o desenvolvimento e aplicação de métodos contínuos para avaliação da qualidade dos serviços prestados à saúde é uma prioridade de pesquisa ${ }^{(3,7)}$.

Em virtude da escassez de um instrumento específico para avaliação da qualidade de um serviço denominado Time de Resposta Rápida (TRR), cujo objetivo é o atendimento imediato do paciente que se torna crítico na unidade de internação, ou seja, ambiente externo à unidade de terapia intensiva ${ }^{(8)}$, o objetivo desta pesquisa foi o de elaborar um instrumento para avaliação da qualidade dos aspectos inerentes a percepção do enfermeiro quanto à estrutura, ao processo e ao resultado, dos serviços prestados pelo Time de Resposta Rápida em um Hospital Universitário Público.

\section{METODOLOGIA}

Trata-se de uma pesquisa exploratória e descritiva, de abordagem quantitativa. O estudo foi realizado no Hospital Universitário de Londrina-PR (HUL), órgão suplementar da Universidade Estadual de Londrina (UEL).

Foram convidados para atuar como avaliadores do questionário quinze profissionais, sendo dez enfermeiros e cinco docentes da área da saúde. Para a seleção da amostra de enfermeiros foram utilizados os seguintes critérios: ter nível relevante de experiência profissional de no mínimo cinco anos na área de enfermagem clínica e/ou cirúrgica ou ainda, no cuidado ao paciente crítico adulto e atuar junto à instituição de saúde vinculada ao estudo.

Para a seleção da amostra de docentes foram utilizados os seguintes critérios: atuar há mais de cinco anos nos Cursos de Graduação em Medicina, Enfermagem ou Fisioterapia, do Centro de Ciências da Saúde (CCS) da UEL, e estar inserido em programas de pós-graduação stricto sensu.

O período de coleta de dados ocorreu no mês de março de 2012, sendo divididos em duas etapas. Na primeira etapa foi entregue a proposta do questionário inicial aos enfermeiros e a segunda etapa constituiu na distribuição do questionário após reformulações dos enunciados sugeridos pelo grupo de enfermeiros aos especialistas docentes. O questionário foi acompanhado de um Termo de Consentimento Livre e Esclarecido esclarecendo sobre os objetivos da pesquisa, bem como as atividades solicitadas.

O questionário inicial foi composto por três partes. A primeira parte constava de itens referentes à identificação, área de atuação e qualificação profissional dos especialistas. A segunda parte constava do roteiro, fundamentado no modelo conceitual de Donabedian, com questões sobre a percepção do entrevistado a respeito de indicadores de estrutura, processo e resultado. $\mathrm{O}$ indicador estrutura corresponde aos recursos físicos, humanos, materiais, equipamentos, normas, rotinas, sistema de valores e expectativas; o processo relaciona-se às percepções dos enfermeiros de como a assistência está sendo prestada aos usuários do serviço de saúde, segundo 
padrões técnico-científicos estabelecidos e aceitos cientificamente; o resultado corresponde às consequências das atividades realizadas nos serviços de saúde, ou pelos profissionais envolvidos ${ }^{(4)}$.

O objetivo do questionário foi contemplar as dimensões representativas que mais impactam no atendimento de um Time de Resposta Rápida. As atividades desenvolvidas pelo TRR descritas na literatura e vivência do pesquisador no serviço de urgência/emergência da instituição em estudo subsidiaram a elaboração dos enunciados em cada indicador.

A proposta do questionário inicial contou com oito proposições na dimensão estrutura, quinze em processo e nove proposições em resultado, totalizando trinta e dois enunciados. Foi solicitado ao especialista responder se cada enunciado avaliaria uma característica ou dimensão do indicador pesquisado. A partir do momento em que o especialista julgasse não concordar com o enunciado havia espaço para descrição de sua sugestão, bem como, um campo para construção de novos enunciados nos três indicadores.

A terceira parte do questionário constava de três colunas em branco para descrição das potencialidades, fragilidades e sugestões sobre o serviço em estudo. Os questionários foram entregues, pelo pesquisador, sendo estabelecido um prazo de dez dias para o retorno.

Foi aplicada a técnica Delphi que consiste em uma ferramenta de pesquisa que busca consenso de opiniões de um grupo de especialistas a respeito de um problema complexo, com possibilidade de revisão das percepções individuais acerca do fenômeno estudado, com base em representação estatística. O procedimento para sua aplicação inclui a circulação do questionário, repetidas vezes, por um painel de especialistas. Esta dinâmica confere ao instrumento avaliado a consolidação do julgamento intuitivo dos especialistas. Em geral com duas ou três rodadas entre grupos participantes obtém a finalidade da redução do nível de divergência, de modo que se atinja a previsão do grupo, sem que haja a necessidade de um painel de discussões ${ }^{(9)}$.

Estabelecer o nível de consenso durante a técnica Delphi é tarefa reservada ao pesquisador e deve ser arbitrário e decidido antes da análise dos dados coletados. Para o estabelecimento dos resultados no índice de concordância padronizado no estudo (valor igual ou maior a $80 \%$ ), foram necessárias duas fases da técnica Delphi. O questionário em sua versão final com as contribuições dos especialistas foi submetido à correção da língua portuguesa por docentes do Departamento de Letras da UEL.

A inclusão dos participantes da pesquisa obedeceu à Resolução no 196/96 do Conselho Nacional de Saúde do Ministério da Saúde, que dispõe sobre Diretrizes e Normas regulamentares da pesquisa envolvendo seres humanos. O projeto foi apreciado e aprovado pelo Comitê de Ética em Pesquisa (CEP) do referido hospital, conforme folha de rosto $\mathrm{n}^{\circ}$ 443992, CAAE $\mathrm{n}^{\circ}$. 0184.0.268.268-11 e parecer CEP/ UEL $n^{\circ} 213 / 2011$. Com relação à autorização do sujeito da pesquisa, o mesmo foi obtido por meio do Termo de Consentimento Livre e Esclarecido, sendo garantida a todos a liberdade de participarem ou não e de desistirem no momento que desejassem. Foram assegurados o sigilo dos dados e o anonimato na identificação.

\section{RESULTADOS}

A maioria da amostra foi composta por especialistas do sexo feminino (93,33\%). A faixa etária variou de 31 a 60 anos, observando-se que os enfermeiros apresentaram média de idade de 44,4 anos, com desvio padrão de \pm 5,8 anos, enquanto os docentes apresentaram média de idade de 49,8 anos com desvio padrão de $\pm 6,3$ anos. Verificou-se que $60 \%$ do total de especialistas possuíam o título de doutor e, quanto ao tempo de experiência profissional, $60 \%$ da amostra apresentavam entre 21 e 35 anos. O grupo apresentou índice elevado de tempo profissional e forneceu contribuições valiosas e criteriosas para a elaboração do instrumento. Todos os 15 especialistas que compuseram a amostra devolveram os questionários no prazo determinado.

\section{APRECIAÇÃO DOS ESPECIALISTAS}

A técnica Delphi foi aplicada em duas fases para se alcançar consenso sobre a estrutura e o conteúdo da segunda versão do instrumento.

\section{Fase Delphi 1}

Quanto ao indicador estrutura, dos oito enunciados avaliados pelo grupo de especialistas enfermeiros, seis $(75 \%)$ sofreram reformulações quanto a ideia/estrutura e apenas dois (25\%) alcançaram índice de concordância $\geq 80 \%$. Foi acrescido um enunciado nesta categoria que foi submetido a avaliação posteriormente pelo segundo grupo de especialistas. A menor concordância foi encontrada no item $n^{\circ} .4$ com percentuais de $30 \%$ e versava sobre os recursos humanos na equipe de enfermagem para prestar o cuidado ao paciente atendido pelo Time de Resposta Rápida. Os autores deste trabalho chamam a atenção que devido à implantação do serviço de resposta rápida em hospitais constituir um serviço relativamente novo no Brasil, ainda não dispõe de um dimensionamento descrito por parte dos órgãos fiscalizadores de enfermagem para quantificar o trabalho e horas necessárias para os cuidados prestados ao paciente crítico atendido por este serviço. O percentual de concordância variou de $30 \%$ a $80 \%$ nesta dimensão.

Quanto ao indicador processo, houve 15 enunciados, dos quais $11(73,33 \%)$ alcançaram o índice de concordância padronizado pelos autores; quatro $(26,67 \%)$ sofreram reformulações e houve a adição de cinco novos enunciados.

Quanto ao indicador resultado, inicialmente constavam nove enunciados, dos quais três $(37,50 \%)$ atingiram índices de concordância proposto, cinco $(62,50 \%)$ sofreram reformulações e houve a exclusão de um enunciado por não estar em concordância com a atividade desenvolvida segundo protocolo de atendimento do Time de Resposta Rápida. O menor índice de concordância neste indicador foi o item $\mathrm{n}^{\circ}$. 2, que abordava se o enfermeiro da unidade temia receber críticas pela equipe e/coordenação do serviço após acionar o serviço 
para o atendimento do paciente que não atendia aos critérios estabelecidos pelo protocolo da instituição.

\section{Fase Delphi 2}

As apreciações dos especialistas na fase 1 embasaram a adequação da segunda versão do questionário, constituído agora por 37 enunciados, ante 32 da proposta inicial. A seguir, foi iniciada a segunda fase, em que foi entregue o questionário ao grupo de especialistas docentes. Após a devolução e tabulação dos questionários houve um índice de concordância de $91 \%$ para o indicador estrutura, 98\% para processo e $95 \%$ para o indicador resultado. Nesta etapa não houve adição ou exclusão de qualquer item nos três indicadores. Valores elevados de concordância revelam que a pesquisa embasou a consolidação do questionário, excluindo a necessidade de uma terceira fase devido ao consenso ser alcançado.

Em síntese, o questionário inicial proposto pelos autores ao final da apreciação pelos especialistas enfermeiros (Grupo 1) demonstrou índices de concordância inferiores ao padronizado na pesquisa, no que referia aos indicadores estrutura e resultado. Após a avaliação pelos enfermeiros, o instrumento foi submetido a um novo grupo de especialistas (Grupo 2), pois optou-se por trabalhar com grupos heterogêneos de profissionais para aumentar o rigor da avaliação. Em virtude desta ação, a obtenção dos índices ao término da técnica Delphi foram satisfatórios para a consolidação do instrumento.

Ressalta-se que mesmo obtendo $84 \%$ de índice de concordância no indicador processo durante a fase Delphi 1, os autores também submeteram este indicador ao Grupo 2, constatando-se melhora significativa deste valor, conforme evidenciado na Tabela 1.

Tabela 1 - Distribuição percentual dos índices de concordância dos especialistas segundo elementos do modelo conceitual de Donabedian, aplicados ao instrumento de avaliação da qualidade do Time de Resposta Rápida, Londrina-PR, 2013

\begin{tabular}{lccc}
\hline \multirow{2}{*}{ Especialistas } & \multicolumn{3}{c}{ \% de Concordância } \\
& Estrutura & Processo & Resultado \\
\hline $\begin{array}{c}\text { Grupo 1 - Enfermeiros } \\
\text { (Fase 1) }\end{array}$ & 65 & 84 & 62 \\
$\begin{array}{c}\text { Grupo 2 - Docentes } \\
\text { (Fase 2) }\end{array}$ & 91 & 98 & 95 \\
\hline
\end{tabular}

\section{DISCUSSÃO}

O questionário elaborado (Apêndice A), considerado em sua versão final após submissão ao painel de especialistas, obteve uma reestruturação passando a contabilizar trinta e sete enunciados, assim divididos: nove proposições em estrutura, vinte em processo e oito em resultado.

Ao conduzir a análise de conteúdo, o questionário sofreu ajustes significativos, quanto à clareza do enunciado, pertinência e organização das proposições. Ao término do tratamento estatístico obtiveram-se índices de concordância nas três dimensões que variaram entre $91 \%$ a $98 \%$, valores estes superiores ao que padroniza a literatura científica, onde deve haver variações finais entre $50 \%$ a $80 \%{ }^{(9)}$, o que demonstra que o questionário pode ser utilizado medindo com acurácia aquilo que se propôs.

De fato, valores desta ordem de grandeza são considerados altamente satisfatórios para os fins a que estas escalas se destinam. Assim, é previsível que, em contextos institucionais com características semelhantes ao cenário do estudo, o instrumento possa ser utilizado para o levantamento das tendências de qualidade no serviço de saúde.

A apreciação por um painel de especialistas torna o instrumento passível de adições e/ou extrações de enunciados em sua elaboração. $\mathrm{O}$ indicador estrutura obteve índice na fase Delphi 1 de apenas $65 \%$ e $91 \%$ na fase 2, sendo acrescido de uma proposição, totalizando nove enunciados. Pesquisa realizada com 32 farmacêuticos da rede pública da macro e microrregião de Fortaleza-CE, com a finalidade de obter os indicadores adequados à realidade local para avaliação da assistência farmacêutica, utilizando a trilogia de Donabedian e a técnica de consenso Delphi, apontou que houve um aumento de sete indicadores na dimensão estrutura após o término da aplicação da técnica Delphi, totalizado 40 itens $^{(10)}$.

$\mathrm{O}$ indicador processo foi o único da tríade a obter o valor de consenso acima do padronizado pela pesquisa e também o maior número de adições de enunciados ao final do método de consenso, de 15 para 20 itens. Em estudo realizado sobre a construção de indicadores da qualidade do cuidado prestado pela Enfermagem na prevenção de eventos adversos em uma unidade médico-cirúrgica, com o uso da técnica Delphi, encontrou-se resultados semelhantes aos propostos nesta pesquisa, havendo a ampliação de 32 para 49 itens de verificação quanto à qualidade do cuidado de enfermagem ${ }^{(11)}$.

Em pesquisa realizada para validação dos cuidados prescritos por enfermeiros aos pacientes ortopédicos em um hospital da região sul do país, os autores relataram que a técnica Delphi demonstrou ser adequada à proposta de estudo, economicamente viável e possibilitou a participação de profissionais altamente qualificados em uma temática na qual a pesquisa é ainda incipiente ${ }^{(12)}$.

Vale ressaltar ainda, dentro do tema de elaboração de instrumento para avaliação da qualidade de serviços de saúde, outro estudo que relata a construção de um instrumento para mensuração de atitudes dos enfermeiros que realizam avaliações de desempenho profissional, em um Hospital Universitário na cidade de São Paulo, em que os autores afirmam que a finalidade do emprego da construção do instrumento assegura clareza dos enunciados e, ao trabalhar com escalas, os pesquisadores devem preocupar-se em empregá-las bem, pois uma escala confiável deve produzir resultados consistentes e coerentes, proporcionando um diagnóstico local do estudo, além de trazer à tona vários novos questionamentos ${ }^{(13)}$.

O pesquisador ao aplicar o instrumento ao objeto de investigação deve estar atento à confiabilidade e estabilidade do mesmo e demonstrar que, se aplicado novamente à mesma população, apresentará os mesmos resultados, como uma 
balança, apontando o mesmo peso em mensurações diferentes de um mesmo objeto.

Para o indicador resultado, verifica-se que foi o item que melhor pontuou após a aplicação da técnica Delphi, com valor do índice de concordância variando de $62 \%$ para $95 \%$ ao final da análise.

Em estudo realizado em São José do Rio Preto-SP, houve a necessidade de reelaborar um instrumento de classificação de pacientes quanto aos cuidados individualizados ao cliente adulto, pois na fase inicial de elaboração do mesmo o instrumento parecia não retratar a complexidade do paciente. Assim houve a necessidade da reaplicação da técnica Delphi em três fases para posterior aplicação do instrumento, no intuito de demonstrar os resultados de classificação de pacientes, quanto aos conteúdos das áreas de cuidados na nova proposta do instrumento ${ }^{(14)}$.

Estudo realizado no campo da tecnovigilância hospitalar, para a construção de instrumento para a avaliação de produto médico-hospitalar por meio da técnica Delphi, baseou-se na necessidade de se obter um modelo validado para amplo uso nas instituições de saúde e que fosse capaz de subsidiar uma das etapas de aquisição desse produto. $\mathrm{O}$ instrumento desenvolvido na pesquisa foi adequado para avaliar o produto e trouxe a possibilidade de aplicar a metodologia na avaliação de outros produtos médico-hospitalares contribuindo para a qualificação e a segurança na utilização. Os autores enfatizam a importância da criação de instrumento que possa validar esse processo, de forma a verificar precocemente as necessidades de adaptação ou reformulação, de acordo com diferentes realidades que dele se utilizam ${ }^{(15)}$.

Faz-se importante mencionar as limitações deste estudo, entre elas a não inclusão de indicador relativo ao aspecto interativo entre o Time de Resposta Rápida e o paciente na dimensão resultado. Realmente, este é um aspecto extremamente importante, porém, não foi vislumbrado no desenvolvimento deste estudo em razão de que em diversos cenários o paciente encontra-se com seu quadro clínico agravado, por muitas vezes permanecer em ventilação mecânica, com estado neurológico alterado ou apresentando outros sinais e sintomas que o tornava impossibilitado de avaliar sua percepção sobre o serviço que tem como objetivo atender o cliente no estado de urgência clínica ou instabilidade hemodinâmica.

\section{CONCLUSÕES}

Em se tratando de pesquisas avaliativas, a utilidade de criar um instrumento com enunciados referentes à percepção dos enfermeiros sobre a avaliação da estrutura, processo e resultado é sempre um assunto de relevância. Sendo assim, pretendemos contribuir com uma proposta de questionário para a avaliação subjetiva do serviço e, com os resultados, subsidiar a tomada de decisões para o melhor benefício das práticas nas instituições hospitalares. Elaborar um questionário simplificado, objetivo e direcionado às necessidades mais urgentes de intervenção possibilita instrumentalizar os gerentes em razão de que os resultados encontrados funcionam como marcadores da qualidade, os quais subsidiam a elaboração e reajuste de metas na busca pela melhor qualidade possível.

Esperamos que a experiência aqui compartilhada estimule outros grupos de investigação a conduzir e testar suas próprias inovações que venham a contribuir para uma ciência viva e criativa. Cabe destacar, porém, que investigações desta natureza podem requerer outras complementações teóricas, bem como fundamentar discussões que incitem o seu aprimoramento e a submissão a validação do mesmo, pois os instrumentos de avaliação muitas vezes não esgotam todo o arcabouço conceitual subjacente às questões envolvidas na área de qualidade dos serviços de saúde.

\section{REFERÊNCIAS}

1. Laurinavicius AG, Peterlini F. Divulgação do time de resposta rápida como ferramenta para a qualidade: impactos sobre a morbi-mortalidade. In: Anais IX Congresso Internacional de Qualidade em Serviços e Sistemas de Saúde; 2009; São Paulo. São Paulo (BR): Qualihosp; 2009. p. 325-6.

2. Chan PS, Khalid A, Longmore LS, Berg RA, Kosiborod M, Spertus JA. Hospital-wide code rates and mortality before and after implementation of a rapid response team. JAMA [Internet]. 2008 [cited 2012 May 20];300(21):250613. Available from: http://www.ncbi.nlm.nih.gov/ pubmed/19050194

3. Gutierres NA. Qualidade nos serviços de saúde. Rev Digital Banas Qualidade [Internet]. 2008 [acesso em 11 de janeiro de 2013];193(6). Disponível em: http:// www.banasqualidade.com.br/2012/portal/conteudo. asp ? codigo $=10214 \&$ secao $=$ Revista

4. Donabedian A. Formulating criteria and standards. In: Donabedian A. An introduction to quality assurance in health care. Oxford (UK): Oxford University; 2003. p. 60-73.

5. D'Innocenzo M, Adami NP, Cunha ICKO. O movimento pela qualidade nos serviços de saúde e enfermagem. Rev Bras Enferm [Internet]. 2006 [acesso em 20 de maio de 2012];59(1):84-8. Disponível em: www.scielo.br/pdf/ reben/v59n1/a16v59n1.pdf

6. Junior AE, Malik AM. Gestão pública em saúde. Cadernos FGV Projetos. [Internet]. 2007 [acesso em 25 de abril de 2013];2(3):5-11. Disponível em: http://bibliotecadigital.fgv.br/dspace/bitstream/handle/10438/6909/300. pdf? sequence $=1$

7. Bittar OJNV. Indicadores de qualidade e quantidade em saúde. Rev Adm Saúde [Internet]. 2008 [acesso em 20 de maio de 2012];3(12):21-8. Disponível em: http://www. saude.am.gov.br/planeja/doc/indicadores.pdf

8. Richmond R. Rapid response teams. Health Policy Newsletter [Internet]. 2008 [cited 2012 May 20];21(2). Available from: http://jdc.jefferson.edu/hpn/vol21/iss2/3/ 
9. Castro AV, Rezende M. A técnica Delphi e seu uso na pesquisa de enfermagem: revisão bibliográfica. REME Rev Min Enferm [Internet]. 2009 [acesso em 20 de maio de 2012];13(3):429-34. Disponível em: http://bases.bireme. br/cgi-bin/wxislind.exe/iah/online/? IsisScript = iah/iah.xis $\& \mathrm{src}=$ google \&base $=$ BDENF\&lang $=p \&$ nextAction $=\ln \mathrm{k}$ \&exprSearch $=17838 \&$ indexSearch $=$ ID

10. Correia ARF, Mota DM, Arrais PSD, Monteiro MP, Coelho HLL. Definição de indicadores para avaliação da assistência farmacêutica na rede pública de Fortaleza-Ceará (Brasil) baseada em métodos de consenso. Acta Farm Bonaer [Internet]. 2009 [acesso em 20 de maio de 2012];28(3):366-74. Disponível em: http://sedici.unlp. edu.ar/handle/10915/7771

11. Vituri DW, Matsuda LM. Validação de conteúdo de indicadores de qualidade para avaliação do cuidado de enfermagem. Rev Esc Enferm USP [Internet]. 2009 [acesso em 20 de maio de 2012];43(2):429-37. Disponível em: http://www.scielo.br/pdf/reeusp/v43n2/a24v43n2.pdf

12. Almeida MA, Pergher AK, Canto DF. Validação do mapeamento de cuidados prescritos para pacientes ortopédicos à classificação das intervenções de enfermagem. Rev Latinoam Enferm [Internet]. 2010 [acesso em 20 maio 2012];18(1):[8 telas]. Disponível em: http://www.scielo. $\mathrm{br} / \mathrm{pdf} / \mathrm{rlae} / \mathrm{v} 18 \mathrm{n} 1 / \mathrm{pt} \_18 . \mathrm{pdf}$

13. Gonçalves VLM, Leite MMJ. Instrumento para mensuração de atitudes frente ao processo de avaliação de desempenho. Rev Bras Enferm [Internet]. 2005 [acesso em 20 de maio de 2012];58(5):563-7. Disponível em: http://www. scielo.br/pdf/reben/v58n5/a12v58n5.pdf

14. Perroca MG. Desenvolvimento e validação de conteúdo de nova versão de um instrumento para classificação de pacientes. Rev Latinoam Enferm [Internet]. 2010 [acesso em 20 de maio de 2012]:19(1):[09 telas]. Disponível em: http://www.scielo.br/pdf/rlae/v19n1/pt_09.pdf

15. Kuwabara CCT, Évora YDM, Oliveira MMB. Gerenciamento de risco em técnico vigilância: construção e validação de um instrumento de produto médico hospitalar. Rev Latinoam Enferm [Internet]. 2010 [acesso em 20 de maio de 2012];18(5):[09 telas]. Disponível em: http:// www.scielo.br/pdf/rlae/v18n5/pt_15.pdf

\section{APÊNDICE A}

Questionário para Avaliação da Qualidade no Atendimento do Time de Resposta Rápida em Hospital Universitário Público

Parte 1 - Caracterização dos Enfermeiros TRR

\section{Dados complementares}

Idade: (anos)

\section{Gênero:}

( ) 1-Masculino ( ) 2-Feminino

\section{Escolaridade:}

( ) 1-Graduação ( ) 2-Especialização ( ) 3-Mestrado ( ) 4-Doutorado

\section{Campo de atuação profissional:}

( ) 1-Hospitalar

( ) 2-Academia/ensino - graduação/pós-graduação

( ) 3-Ambos (Hospitalar e Academia/ensino)

( ) 4-Outro:

\section{Tempo de atuação profissional no Hospital Universitário:}

( ) 1-Igual ou menor que 5 anos

( ) 2-Entre 6 e 10 anos

$$
\begin{array}{ll}
\text { ) } 2 \text {-Entre } 6 \text { e } 10 \text { anos } & \text { ( ) 3-Entre } 11 \text { e } 15 \text { anos } \\
\text { 4-Entre } 16 \text { e } 20 \text { anos } & \text { ( ) 5-Entre } 21 \text { e } 25 \text { anos }
\end{array}
$$$$
\text { ( ) 6-Entre } 26 \text { e } 30 \text { anos ( ) 7-Mais que } 31 \text { anos }
$$

\section{Unidade de atuação no Hospital Universitário:}

( ) Unidades de Internação: Masculina, Tisiologia, Feminina, Infectocontagiosa

( ) Pronto Socorro 
Parte 2 - Questionário para Avaliação da Qualidade do TRR

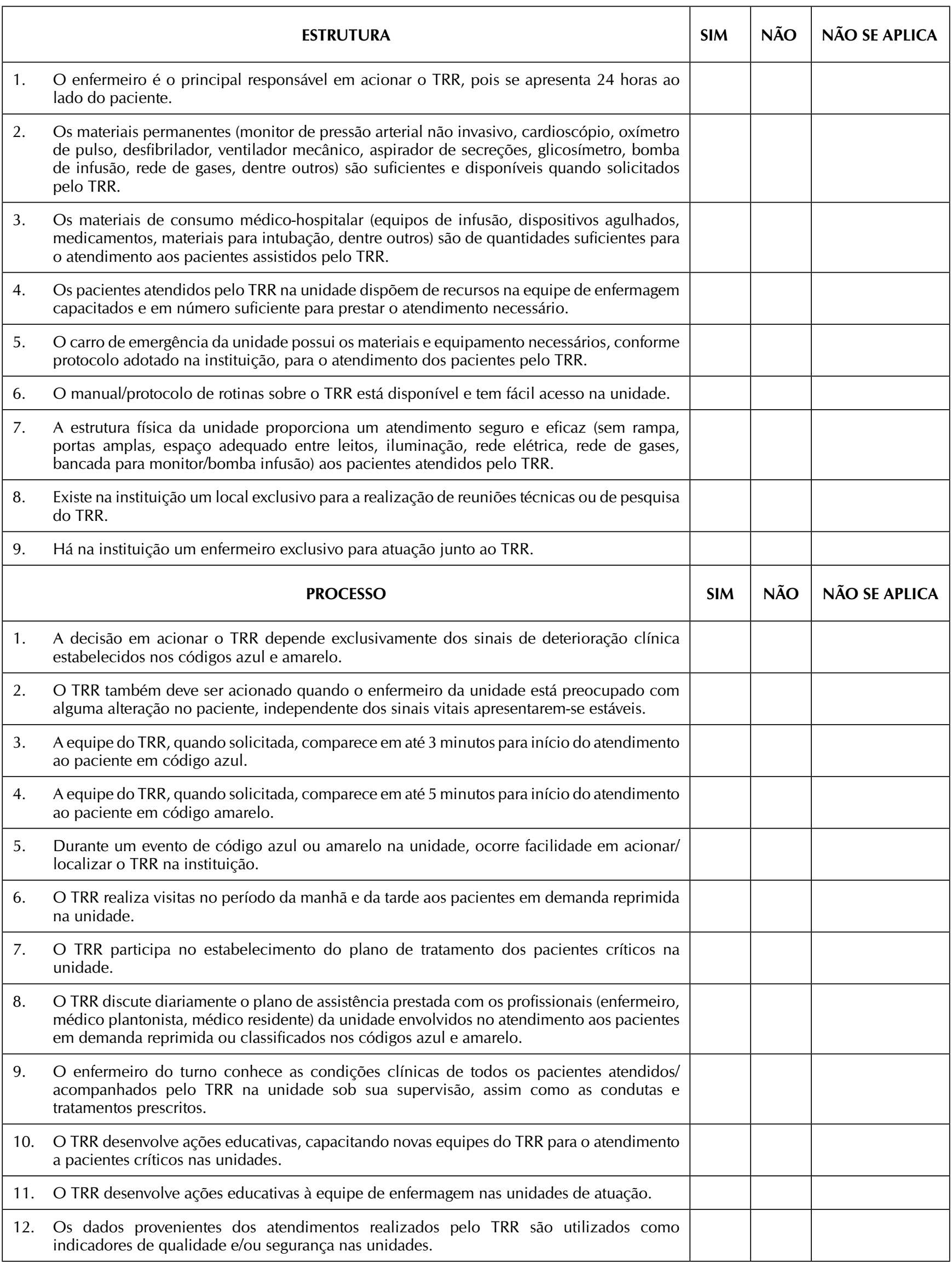




\begin{tabular}{|c|c|c|c|c|}
\hline \multicolumn{5}{|c|}{$\begin{array}{l}\text { 13. O TRR possui grupo de pesquisa/estudo formado pela equipe do TRR e por profissionais } \\
\text { das unidades. }\end{array}$} \\
\hline 14. & $\begin{array}{l}\text { É fundamental o acompanhamento dos pacientes críticos em demanda reprimida pelo TRR, } \\
\text { devido à dificuldade de transferência imediata para a Unidade de Terapia Intensiva. }\end{array}$ & & & \\
\hline 15. & $\begin{array}{l}\text { Ocorre uma comunicação efetiva entre o médico do TRR e o médico da clínica titular nas } \\
\text { condutas sugeridas aos pacientes. }\end{array}$ & & & \\
\hline 16. & O TRR se comunica com o enfermeiro ao realizar visita aos pacientes nas unidades. & & & \\
\hline 17. & A comunicação entre membros do TRR e o enfermeiro da unidade é efetiva, clara e objetiva. & & & \\
\hline 18. & $\begin{array}{l}\text { Os profissionais do TRR são acessíveis para o atendimento dos códigos amarelo e azul e ou } \\
\text { demanda reprimida. }\end{array}$ & & & \\
\hline 19. & $\begin{array}{l}\text { O enfermeiro da unidade acompanha o atendimento do código azul durante o período } \\
\text { total de atendimento, juntamente com os profissionais do TRR. }\end{array}$ & & & \\
\hline 20. & $\begin{array}{l}\text { O enfermeiro da unidade acompanha o atendimento do código amarelo durante o } \\
\text { período total de atendimento, juntamente com os profissionais do TRR. }\end{array}$ & & & \\
\hline & RESULTADO & SIM & NÃO & NÃO SE APLICA \\
\hline 1. & $\begin{array}{l}\text { Com o atendimento do médico titular e do TRR prestado concomitantemente, tenho maior } \\
\text { segurança na execução da assistência de enfermagem prestada na unidade sob minha } \\
\text { supervisão. }\end{array}$ & & & \\
\hline 2. & $\begin{array}{l}\text { Enquanto enfermeiro tenho receio de receber críticas da instituição ou do coordenador } \\
\text { do TRR, ao acionar a equipe do serviço, quando os pacientes não atendem aos critérios } \\
\text { estabelecidos pelo protocolo. }\end{array}$ & & & \\
\hline 3. & $\begin{array}{l}\text { O atendimento diferencial proposto pelo TRR ao paciente em demanda reprimida e/ou } \\
\text { acompanhado por } 72 \text { horas após a alta da Unidade de Terapia Intensiva melhora a assistência } \\
\text { prestada aos pacientes. }\end{array}$ & & & \\
\hline & É fundamental implantar o atendimento do TRR no período noturno na instituição. & & & \\
\hline 5. & A atuação do TRR reduz o risco de deterioração clínica dos pacientes nas unidades. & & & \\
\hline & $\begin{array}{l}\text { A atuação do TRR reduz a taxa de mortalidade dos pacientes em código azul, amarelo ou } \\
\text { demanda reprimida. }\end{array}$ & & & \\
\hline & $\begin{array}{l}\text { O TRR e a equipe de enfermagem da unidade sob minha supervisão possuem um bom } \\
\text { relacionamento interpessoal. }\end{array}$ & & & \\
\hline & $\begin{array}{l}\text { A instituição ou o coordenador do TRR fornecem mensalmente os resultados dos } \\
\text { atendimentos realizados nas unidades. }\end{array}$ & & & \\
\hline
\end{tabular}

Parte 3 - Considerando sua experiência na Unidade com o TRR, aponte potencialidades, fragilidades e sugestões sobre o atendimento do TRR

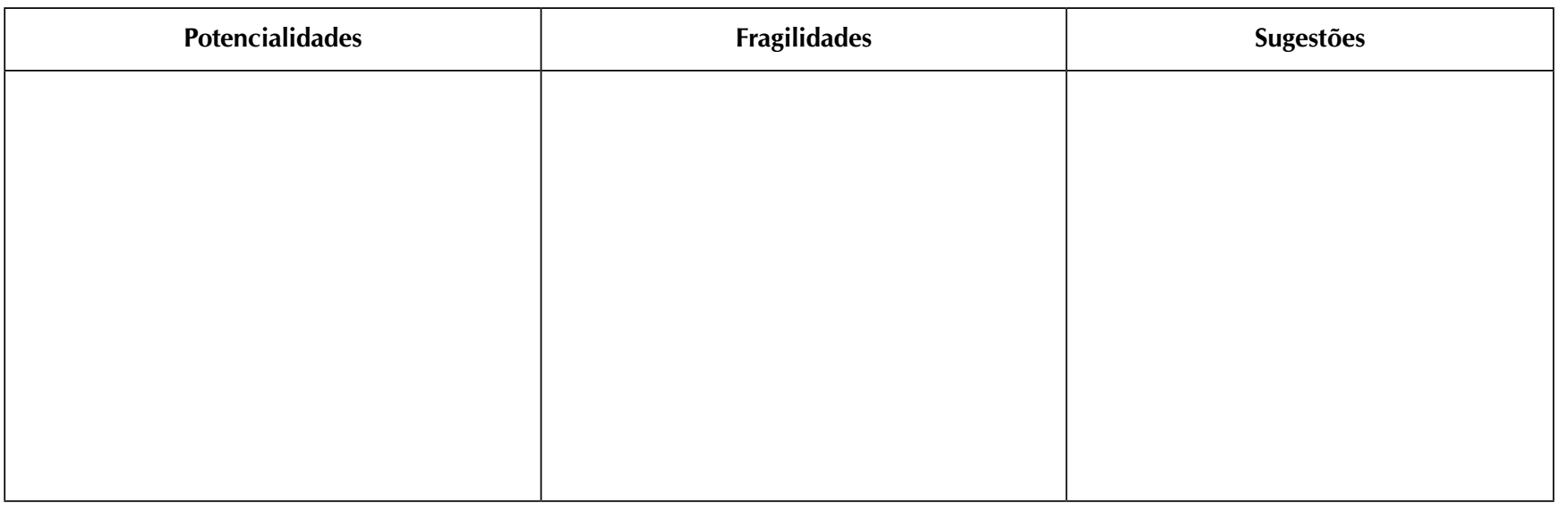

